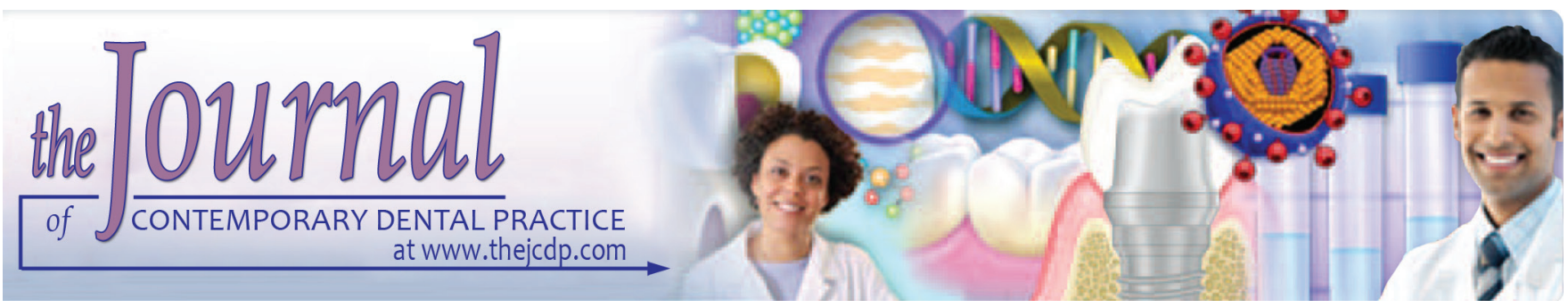

\title{
Effect of Addition of Platelet-rich Plasma to Calcium Phosphosilicate Putty on Healing at 9 Months in Periodontal Intrabony Defects
}

\author{
${ }^{1}$ Sagrika Shukla, ${ }^{2}$ Ashi Chug, ${ }^{3}$ Lanka Mahesh, ${ }^{4}$ Harpreet Singh Grover
}

\begin{abstract}
Background and objective: Combination of platelet-rich plasma (PRP) and bone substitutes for the surgical treatment of periodontal intrabony defects is based on a sound biologic rationale; however, the clinical results indicative of the synergistic effect of PRP remain ambiguous. The objective of the present study was to clinically and radiographically evaluate the use of calcium phosphosilicate (CPS) putty alone and in combination with PRP in the treatment of periodontal intrabony defects.
\end{abstract}

Materials and methods: The study was performed at an outpatient facility at a teaching dental institute in north India. A split-mouth design was employed to assess the clinical parameters and radiographic bone fill following the use of CPS putty with and without PRP in patients scheduled for surgical periodontal treatment of intrabony osseous defects. Each defect was randomized to receive treatment with open flap debridement, with CPS putty alone (Group PUT), or open flap debridement with CPS putty and PRP (Group PRP). Probing pocket depth (PPD), plaque index $(\mathrm{PI})$, gingival index $(\mathrm{GI})$, and clinical attachment levels (CALs) were recorded at the investigated sites utilizing custom-made reference guides for measurement reproducibility. Standardized periapical radiographs were also obtained to evaluate defect fill at the surgical sites.

Results: Twenty patients each with at least two defects located in different quadrants were enrolled. The reduction in PPD from baseline to 1st, 3rd, 6th, and 9th month was found to be significant $(p<0.05)$. The percent reduction in PPD among PUT group was $57.18 \pm 10.71 \%$ and among PRP group was $51.39 \pm 12.60 \%$. No

\footnotetext{
${ }^{1}$ Department of Periodontology, Seema Dental College and Hospital, Rishikesh, Uttarakhand, India

${ }^{2}$ Department of Dentistry and Oral and Maxillofacial Surgery All India Institute of Medical Sciences, Rishikesh, Uttarakhand, India

${ }^{3}$ Private Practice, The Specialist Clinic, Saket, New Delhi, India ${ }^{4}$ Department of Periodontology, Faculty and Dental Sciences SGT Dental College and Hospital, SGT University, Gurgaon Haryana, India

Corresponding Author: Sagrika Shukla, Faculty, c-488 SFS Flats, Sheikh Sarai Phase - I, New Delhi -110017, India Phone:+91-9873090671, e-mail: Shukla.sagrika@gmail.com
}

statistically significant difference was observed in the percent reduction in PPD among two groups at 9 months $(p=0.48)$. Sites in both groups exhibited statistically significant reductions in $\mathrm{PI}$ and $\mathrm{Gl}$ that were maintained throughout the study period. Similar results were seen while measuring CAL.

Conclusion: Calcium phosphosilicate Putty alone provides significant improvement in outcomes for the treatment of periodontal intraosseous defects. The addition of PRP to CPS putty does not seem to provide any additive benefit to treatment and the additional surgical time and trauma can be avoided.

Keywords: Intrabony defects, Novabone ${ }^{\circledR}$ bone graft, Plateletrich plasma (PRP), Regeneration.

How to cite this article: Shukla S, Chug A, Mahesh L, Grover HS. Effect of Addition of Platelet-rich Plasma to Calcium Phosphosilicate Putty on Healing at 9 Months in Periodontal Intrabony Defects. J Contemp Dent Pract 2016;17(3):230-234.

Source of support: Partial material support was given by Dr Srinivas Katta, Director - Dental Products, (Alachua, FL) to Dr George A. Kotsakis DDS, Advanced Education Program in Periodontology, University of Minnesota and Dr Bhanu K. Bhakri MD (Pediatrics), All India Institute of Medical Sciences for kind support and guidance.

Conflict of interest: All of the authors on this research report no financial relationships related to any products listed in this study.

\section{INTRODUCTION}

The treatment of intrabony defects frequently results in favorable improvement in the prognosis of an affected tooth due to their increased regenerative potential in comparison to areas of horizontal bone loss. ${ }^{1,2}$ Surgical treatment of these types of defects frequently involves either bone grafts or alloplastic bone substitutes along with the use of barrier membranes or growth factors to increase the probability of bone regeneration in the area. ${ }^{1,2}$ Calcium phosphosilicate putty (CPS putty) (NovaBone 
Dental Putty; NovaBone Products, Alachua, FL), a commonly used alloplastic bone substitute, has been observed to have an osteostimulative effect in addition to osteoconductive properties and enhanced handling characteristics. ${ }^{3,4}$ Several authors have proposed the addition of autologous growth factors such as plateletrich plasma (PRP) to be a safe measure to enhance the results of graft placement. ${ }^{5,6}$ Platelet-rich plasma contains proinflammatory cytokines, growth factors, and other factors such as serotonin, histamine, dopamine, calcium, and adenosine, which have fundamental effects on the biologic aspects of wound healing. ${ }^{7}$ However, the superior clinical results from its addition to the bone substitute are still debated.

The aim of this study was to evaluate the clinical efficacy of CPS putty alone, or in combination with PRP in the treatment of periodontal intrabony defects.

\section{MATERIALS AND METHODS}

The study was conducted for a total period of 3 years at outpatient services of a teaching dental institute in north India. Split-mouth design evaluation was performed to evaluate the additive effect of CPS putty along with PRP on the treatment of intrabony periodontal defects. Twenty prospective adult patients presenting with at least two intrabony defects located in different quadrants, with residual probing pocket depth of $>5 \mathrm{~mm}$ and radiographic defect height $>3 \mathrm{~mm}$, were screened for enrolment in the study to be evaluated clinically and radiographically for 9 months. Pregnant females, smokers, and those allergic to CPS were excluded. In addition, those with poor oral hygiene or having systemic illness and drug exposures likely to affect surgical outcomes and healing were also excluded. The eligible candidates were offered participation in the study and written consent was obtained from those finally enrolled. The flow of study participants from initial screening to final analysis is summarized in Flow Chart 1.

\section{Assessment}

All the evaluations were performed by an independent trained observer, not involved in the study. The clinical parameters, viz. pocket probing depth (PPD), clinical attachment levels (CAL), plaque index $(\mathrm{PI}),{ }^{8}$ and gingival index $(\mathrm{GI})^{9}$ were recorded using recommended procedure (Fig. 1 -right). These parameters were recorded at baseline and then at 3 monthly intervals till 9 months of follow-up. The percent change in PPD at 9 months following treatment was taken as primary outcome measure, compared between the two groups. Radiographic assessment included digital intraoral periapical radiographs taken with the aid of a positioning device for standardization of preoperative and postoperative radiographs (Fig. 1 - left).
Flow Chart 1: The study participants from enrollment to final analysis
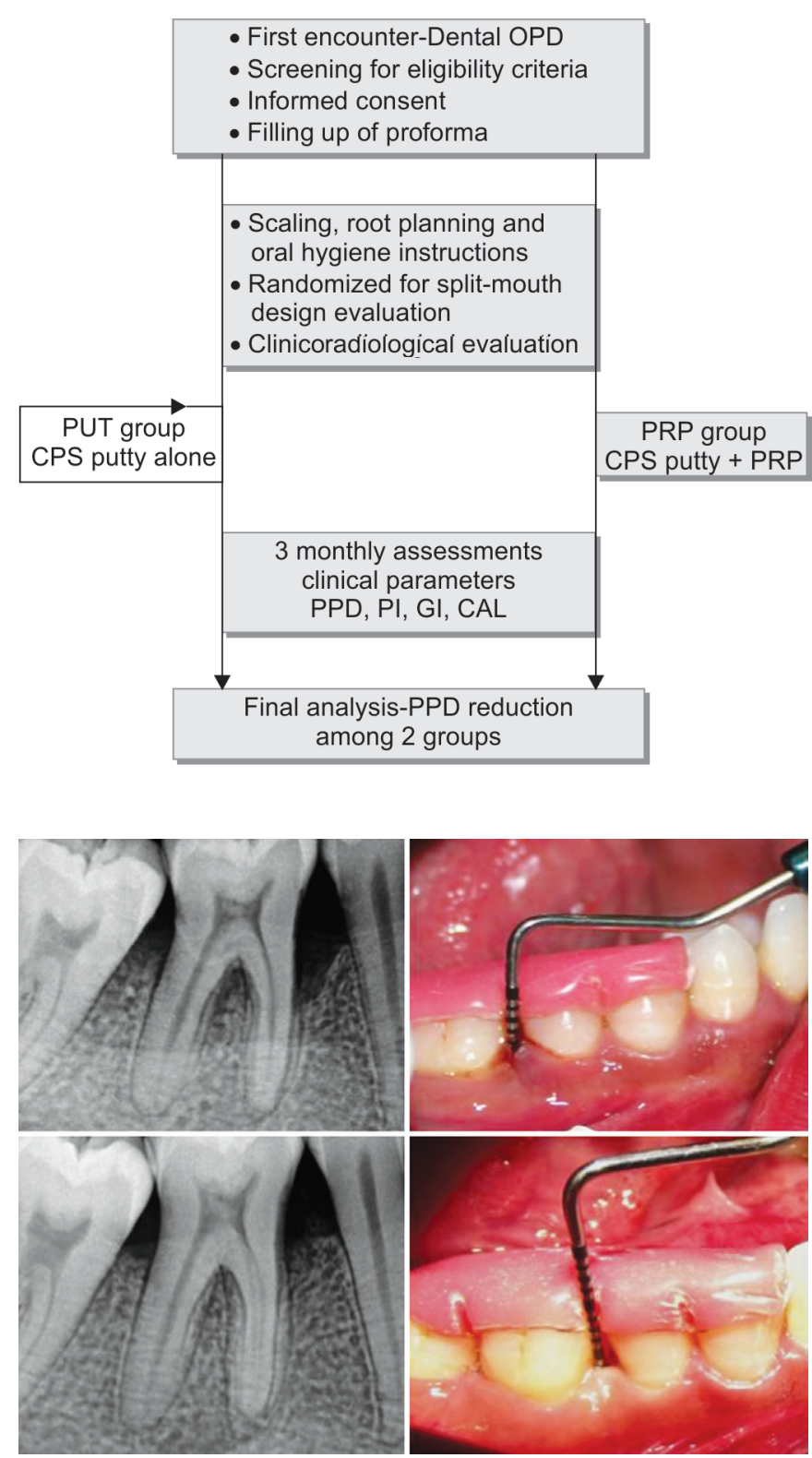

Fig. 1: Clinical (right) and radiological (left) assessment performed over sites at baseline (upper row) and at 9 months postoperative (lower row)

During assessment, the cementoenamel junction (CEJ) and the base of the defect (BD) were located on the digital periapical image and bone fill assessment was done by recognizing the defect in the preoperative and postoperative radiographs and assessing the gain in bone height.

\section{Treatment}

Each of the enrolled patients underwent scaling and root planning and received chair side oral hygiene instructions, as a prerequisite, 4 weeks prior to initial evaluation. Following re-evaluation, oral hygiene instructions were reviewed and each defect was randomized to receive 
treatment with open flap debridement and CPS Putty alone (Group PUT), or open flap debridement with CPS Putty and PRP (Group PRP). Randomization was performed using a computer-generated randomization list. All periodontal procedures were performed on an outpatient basis under aseptic conditions. Standardized surgical procedure was followed for debridement and graft placement for both the groups.

In PRP group, in addition, $10 \mathrm{ml}$ of patient's own blood was drawn prior to the surgical procedure. Blood was mixed with anticoagulant (ethylenediaminetetraacetic acid) and centrifuged for 15 minutes at $2000 \mathrm{rpm}$. After the first spin, three distinct layers were obtained of which the first two layers were pipetted out and placed in a plastic blood collection tube without anticoagulant and were centrifuged at $3000 \mathrm{rpm}$ for 15 minutes. The lower layer was pipetted out and was activated by calcium chloride and the patient's own blood to form PRP. Within a few seconds, the PRP preparation obtained a sticky gel consistency that was mixed with CPS putty that was expressed from the cartridges into a tray and placed into the bony defect. Both the groups received identical standard postoperative instructions, including antibiotics, analgesics, antiseptic rinse, and abstinence from brushing and flossing around surgical area.

\section{Sample Size and Statistical Analysis}

Considering an estimated difference of $0.5 \mathrm{~mm}$ in pocket depth reduction with $0.7 \mathrm{~mm}$ standard deviation, as reported by Leknes et $\mathrm{al}^{10}{ }^{10} 20$ patients were planned to be enrolled, with two defects, each randomized into two groups, with $5 \%$ level of significance and $85 \%$ power. Values of various indices were depicted as Mean \pm SD and compared using paired t-test. The " $p$ " value of $<0.05$ was considered significant. Data were analyzed using SPSS version 16.0 for Windows. The study protocol was approved by institute ethics review committee.

\section{RESULTS}

A total of 20 patients ( 7 female and 13 male, mean age $40 \pm 10.5$ years) completed the 9 -month follow-up as per study protocol. In general, no major adverse events were reported among groups with most patients reporting uneventful healing with mild postoperative discomfort and edema. The baseline clinical characteristics of sites in both the groups were comparable in terms of PPD $(p=0.79)$, GI $(p=0.99), P I(p=0.99)$, and CAL $(p=0.11)$ (Table 1). Mean baseline PPD for the PUT group was $6.95 \pm 1.28 \mathrm{~mm}$ and for the PRP group was $7.05 \pm 1.15 \mathrm{~mm}$. There was a significant reduction in PPD over 9-month follow-up in both the groups (Graph 1). The reduction in PPD from baseline to 1st, 3rd, 6th, and 9th month was found to be significant $(p<0.05)$. The percent reduction in PPD among PUT group was $57.18 \pm 10.71 \%$ and among PRP group was $51.39 \pm 12.60 \%$. No statistically significant difference was observed in the percent reduction in PPD among two groups at 9 months ( $\mathrm{p}=0.48)$.

Sites in both groups exhibited statistically significant reduction in PI, GI that were maintained throughout the study period. The pattern noted in CAL measurements was similar to that noted in PPD for both groups. The PUT and PRP groups had a gain in CAL levels in the range of $5.0 \pm 0.82 \mathrm{~mm}$ and $5.62 \pm 0.74 \mathrm{~mm}$ at 9 months postsurgery respectively. Radiographically, all sites showed radiopaque tissue with trabecular bone patterns in the defects at 9 months postgraft placement indicative of new bone formation (Fig. 1 lower row). The intragroup differences in defect depth were highly statistically significant $(p<0.001)$, while the intergroup difference was not statistically significant $(\mathrm{p}>0.05)$.

Table 1: Clinical outcome measures among the study groups, at baseline, and at 9 months

\begin{tabular}{|c|c|c|c|c|}
\hline & \multicolumn{2}{|c|}{ PUT group: Mean (SD) } & \multicolumn{2}{|c|}{ PRP group: Mean (SD) } \\
\hline & Baseline & 9 months & Baseline & 9 months \\
\hline $\begin{array}{l}\text { Pocket } \\
\text { probing depth } \\
\text { (PPD) (mm) }\end{array}$ & $6.95 \pm 1.27$ & $2.90 \pm 0.55$ & $7.05 \pm 1.14$ & $3.35 \pm 0.74$ \\
\hline $\begin{array}{l}\text { Gingival } \\
\text { index (GI) }\end{array}$ & $1.33 \pm 0.39$ & $1.10 \pm 0.31$ & $1.33 \pm 0.39$ & $1.11 \pm 0.31$ \\
\hline $\begin{array}{l}\text { Plaque } \\
\text { index (PI) }\end{array}$ & $1.74 \pm 0.34$ & $1.46 \pm 0.50$ & $1.74 \pm 0.34$ & $1.64 \pm 0.48$ \\
\hline $\begin{array}{l}\text { Clinical } \\
\text { attachment } \\
\text { level (CAL) }\end{array}$ & $7.40 \pm 1.64$ & $2.40 \pm 0.50$ & $8.25 \pm 1.68$ & $2.63 \pm 0.60$ \\
\hline
\end{tabular}

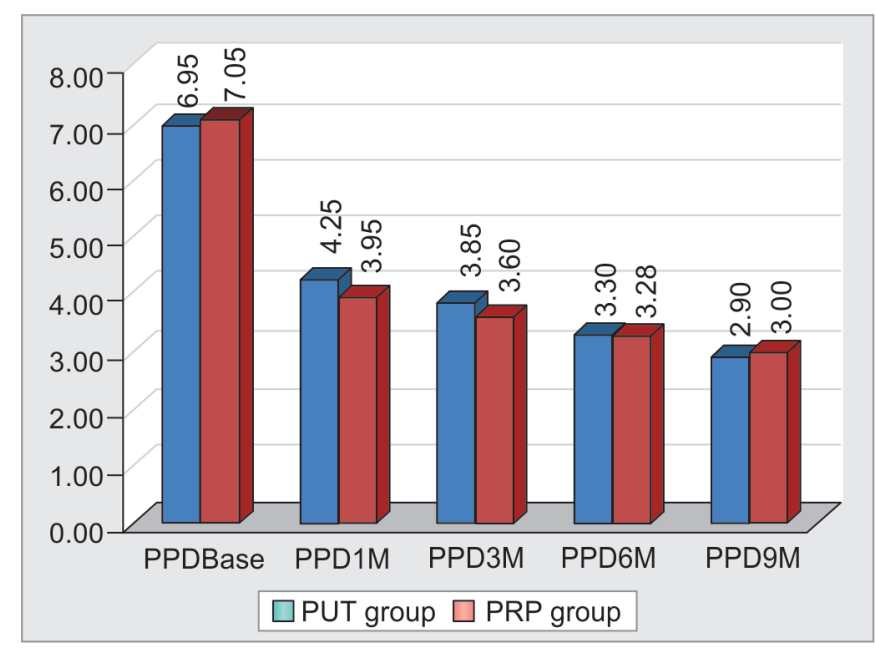

Graph 1: Comparison of pocket probing depth $(\mathrm{mm})$ at baseline, 1, 3, 6, and 9 months 


\section{DISCUSSION}

Our observations clearly indicate that there is no added advantage of adding PRP to the CPS putty graft for treatment of periodontal intrabony defects, in terms of healing at 9 months after procedure. Additionally, radiographic evidence of bone fill were noted in both groups.

Results of this study are directly comparable to the previous work of Froum et $\mathrm{al}^{11}$ and Lovelace et $\mathrm{al}^{12}$ who showed very favorable clinical and radiographic results following the use of bioactive glass in periodontal osseous defects that were superior to those of human allografts. The particulate that forms the active ingredient in CPS Putty has been designed to activate genes that stimulate regeneration of living tissues. ${ }^{13}$ In vivo studies based on this concept have confirmed the higher bone regenerative potential of bioactive glasses, than glass ceramics and hydroxyapatite. ${ }^{14}$ Xynos et $\mathrm{al}^{15,16}$ reported that the ionic products of the glass dissolution affected the geneexpression profile causing upregulation of seven families of genes, including cell cycle regulators, growth-related gene, and apoptosis regulators. Expression of a potent osteoblast mitogenic growth factor, insulin-like growth factor II (IGF-II), was increased to $290 \%{ }^{14}$

These osteostimulative properties have established CPS as a successful material for use in the surgical treatment of periodontal defects. ${ }^{17}$ Other studies by Ong et al, ${ }^{18}$ Park et al, ${ }^{19}$ and Zamet et al $^{20}$ have also demonstrated statistically significant improvements in periodontal indices over a period of 6 months in bioactive glass treated sites. Even though no histological analysis was performed in the present study it is reasonable to hypothesize that the significant reduction in probing pocket depth following the use of CPS putty can be attributed to soft and hard tissue improvements following resolution of inflammation and to the osteogenic potential of the CPS putty used in the study.

An objective of this study was to evaluate the combined effect of CPS putty and PRP in periodontal intrabony defects. The use of PRP for bone augmentation is based on the premise that the large number of platelets in PRP release significant quantities of growth factors, which promote neovascularization and osseous regeneration. ${ }^{21,22}$ Platelet-rich plasma contains a number of different growth factors such as platelet-derived growth factor (PDGF), transforming growth factor beta 1 (TGF $\beta-1)$, Insulin-like growth factor (IGF), epidermal growth factor (EGF) epithelial cell growth factor (ECGF), and also proteins known to act as cell adhesion molecules for osteocindction and as a matrix for bone, connective tissue, and epithelial cell migration. ${ }^{23,24}$ Platelet-rich plasmas stimulate the proliferation of several cell types; however, contrasting results have also been reported, probably due to the great number of PRP protocols that might lead to significantly different platelet concentrates. ${ }^{25,26}$ Apart from an anti-infectious effect, leukocytes produce large amounts of vascular endothelial growth factor (VEGF). ${ }^{27}$ Thus, additional VEGF in PRP preparation, which stems from the leukocytes, might be crucially important for the promotion of angiogenesis. However, the respective effects of platelets and leukocytes in the platelet concentrates have not yet been analyzed, and the contribution of the leukocytes effect remains unclear, such synergistic effects can only be hypothesized. ${ }^{25}$

In the present study, potential benefit of adding PRP was not identified. These results are in accordance with those by Choi et $\mathrm{al}^{28}$ and Schlegel et $\mathrm{al}^{29}$ where addition of PRP did not appear to enhance new bone formation while using autogenous bone grafts for bone regeneration. A possible explanation may also be that PRP does not act synergistically with CPS putty. The osteoconductive and osteostimulative properties of CPS biomaterials are based on intimate contact with the bone that allows the formation of the Si-rich layer that is a crucial stage in bone bonding, as it acts as a template for calcium phosphate precipitation. The calcium phosphate layer then directs new bone formation together with absorbing proteins. ${ }^{30}$ However, when CPS putty and PRP are mixed, due to the heterogeneity of the mix, either PRP or CPS putty will bond with the bone first. Consequently, as PRP and CPS putty compete for intimate bone contact, if PRP bonds first then the CPS putty will not be able to interact with bone, and thus, the bone regeneration potential of the graft will not be combined with the increased healing potential of the PRP.

Although our observations provide useful information on the issue, slight caution must be exercised while generalizing our results to the population at large. The limitations of our study must be considered while interpreting our results. Firstly, absence of an objectively measurable outcome allows some element of subjectivity while taking measurements of clinical parameters, and secondly, due to ethical reasons, no re-entry for tissue specimen collection was performed; therefore, the question of whether the radiographically observed radiopaque tissue was vital bone remains unanswered.

\section{CONCLUSION}

Within the limitations of this study, it can be concluded that CPS Putty alone provides significant improvement in clinical and radiographic outcomes for the treatment of periodontal intraosseous defects. The addition of PRP to CPS putty provided no additive effect. Further 
randomized clinical studies are required to histologically substantiate the benefits of CPS putty in the surgical treatment of periodontal osseous defects.

\section{REFERENCES}

1. Nevins M, Giannobile WV, McGuire MK, Kao RT, Mellonig JT, Hinrichs JE, McAllister BS, Murphy KS, McClain PK, Nevins ML, et al. Platelet-derived growth factor stimulates bone fill and rate of attachment level gain: results of a large multicenter randomized controlled trial. J Periodontol 2005 Dec;76(12):2205-2215.

2. Kotsakis, G, Chrepa, V, Katta, S. Practical application of the newly introduced natural bone regeneration (NBR) concept utilizing alloplastic Putty. Int J Oral Implantol Clin Res 2011;2:145-149.

3. Mahesh L, Kotsakis GA, Venkataraman N, Shukla S, Prasad H. Alveolar ridge preservation with the socket-plug technique utilizing an alloplastic putty bone substitute or a particulate xenograft: a histological pilot study. J Oral Implantol 2015 Apr;41(2):178-183.

4. Mazor Z, Ioannou A, Venkataraman N, Kotsakis G. A minimally invasive sinus augmentation technique using a novel bone graft delivery system. Int J Oral Implantol Clin Res 2013;4:78-82.

5. Nevins M, Giannobile WV, McGuire MK, Kao RT, Mellonig JT, Hinrichs JE, et al. Platelet-derived growth factor stimulates bone fill and rate of attachment level gain: results of a large multicenter randomized controlled trial. J Periodontol 2005 Dec;76(12):2205-2215.

6. Cetinkaya BO, Keles GC, Pamuk F, Balli U, Keles ZP. Longterm clinical results on the use of platelet concentrate in the treatment of intrabony periodontal defects. Acta Odontol Scand 2014 Feb;72(2):92-98.

7. Kim DM, Nevins M, Camelo M, Nevins ML, Schupbach P, Rodrigues VS, Fiorellini JP. Human histologic evaluation of the use of the dental putty for bone formation in the maxillary sinus: case series. J Oral Implantol 2012 Aug;38(4): 391-398.

8. Silness J, Loe H. Periodontol disease is pregnancy and co-relation between oral hygiene and periodontal conditions. Acta Odontol Scand 1964;24:747-759.

9. Loe H, Silness J. Periodontal diseases in pregnancy. Prevalence and severity. Acta Odontol Scand 1963 Dec;21:533-551.

10. Leknes KN, Andersen KM, Bøe OE, Skavland RJ, Albandar JM. Enamel matrix derivative versus bioactive ceramic filler in the treatment of intrabony defects: 12 -month results. J Periodontol 2009 Feb;80(2):219-227.

11. Froum SJ, Weinberg MA, Tarnow D. Synthetic bone graft particles and open debridement in the treatment of human periodontal defects. A clinical study. J Periodontol 1998 Jun:69(6):698-709.

12. LovelaceTB,MellonigJT,MeffertRM,JonesAA,NummikoskiPV, Cochran DL. Clinical evaluation of bioactive glass in the treatment of periodontal osseous defects in humans. J Periodontol 1998 Sep;69(9):1027-1035.

13. Hench LL. The story of bioglass. J Mater Sci Mater Med 2006 Nov;17(11):967-978.

14. Oonishi H,Hench LL, Wilson J, Sugihara F, Tsuji E, Matsuura M, Kin S, Yamamoto T, Mizokawa S. Quantitative comparison of bone growth behavior in granules of Bioglass, A-W glass-ceramic, and hydroxyapatite. J Biomed Mater Res 2000 Jul;51(1):37-46.

15. Xynos ID, Edgar AI, Buttery LDK, Hench LL, Polak JM. Geneexpression profiling of human osteoblasts following treatment with the ionic products of Bioglass $45 \mathrm{~S} 5$ dissolution. J Biomed Mater Res 2001 May;55(2):151-157.

16. Xynos ID, Edgar AI, Buttery LDK, Hench LL, Polak JM. Ionic products of bioactive glass dissolution increase proliferation of human osteoblasts and induce insulin-like growth factor II mRNA expression and protein synthesis. Biochem Biophys Res Commun 2000 Sep 24;276(2):461-465.

17. Sohrabi K, Saraiya V, Laage TA, Harris M, Blieden M, Karimbux N. An evaluation of bioactiveglass in the treatment of periodontal defects: a meta-analysis of randomized controlled clinical trials. J Periodontol 2012 Apr;83(4):453-464.

18. Ong MA, Eber RM, Korsnes MI, Macneil RL, Glickman GN, Shyr Y, Wang HL. Evaluation of a bioactive glass alloplast in treating periodontal intrabony defects. J Periodontol 1998 Dec;69(12):1346-1354.

19. Park JS, Suh JJ, Choi SH, Moon IS, Cho KS, Kim CK, Chai JK. Effects of pretreatment clinical parameters on bioactive glass implantation in intrabony periodontal defects. J Periodontol 2001 Jun;72(6):730-740.

20. Zamet JS, Darbar UR, Griffiths GS. Particulate bioglass as a grafting material in the treatment of periodontal intrabony defects. J Clin Periodontol 1997 Jun;24(6):410-418.

21. Marx RE, Carlson ER, Eichstaedt RM, Schimmele SR, Strauss JE, Goergeff KR. Platelet-rich plasma: growth factor enhancement for bone grafts. Oral Surg Oral Med Oral Pathol Oral Radiol Endod 1998 Jun:85(6):638-646.

22. Kassolis JD, Rosen PS, Reynolds SA. Alveolar ridge and sinus augmentation utilizing platelet-rich plasma in combination with freeze dried bone allograft: case series. J Periodontol 2000 Oct;71(10):1654-1661.

23. Marx RE. Platelet-rich plasma: evidence to support its use. J Oral Maxillofac Surg 2004 Apr;62(4):489-496.

24. Ferreira CF, Gomes CC, Filho JS, Granjeiro JM, Simoes $\mathrm{CMO}$, Magini RS. Platelet -rich plasma influence on human osteoblast growth. Clin Oral Implants Res 2005 Aug;16(4):256-460.

25. Cenni E, Ciapetti G, Pagani S, Perut F, Giunti A, Baldini N. Effects of activated platelet concentrates on human primary cultures of fibroblasts and osteoblasts. J Periodontol 2005 Mar;76(3):323-328.

26. Slapnicka J, Fassmann A, Strasak L, Augustin P, Vanek J. Effects of activated and nonactivated platelet-rich plasma on proliferation of human osteoblasts in vitro. J Oral Maxillofac Surg 2008 Feb;66(2):297-301.

27. Werther K, Christensen IJ, Nielsen HJ. Determination of vascular endothelial growth factor (VEGF) in circulating blood: significance of VEGF in various leukocytes and platelets. Scand J Clin Lab Invest 2002;62(5):343-350.

28. Choi BH, Im CJ, Huh JY, Suh JJ, Lee SH. Effect of platelet-rich plasma on bone regeneration in autogenous bone graft. Int J Oral Maxillofac Surg 2004 Jan;33(1):56-59.

29. Schlegel KA, Kloss FR, Schultze-Mosgau S, Nenkam FW, Wiltfang J. Osseous defect regeneration using autogenous bone alone or combined with Biogran or Algipore with and without added thrombocytes. A microradiologic evaluation. Mund Kiefer Gesichtschir 2003 Mar;7(2):112-118.

30. Andre M. Imaging methods in periodontology. Periodontol 2000 2004;34:34-38. 